\title{
Research and application of Hadoop monitoring based on JMX
}

\author{
Rui Gu \\ Suzhou Industrial Park Institute of Services outsourcing, \\ suzhou, china \\ gur@siso.edu.cn
}

\begin{abstract}
In order to use Hadooop monitoring in specific J2EE project, we design and implement a monitor solution that can be used in specific project based on JMX technology standards. At first, this paper analyzed the JMX specification, simply introduced the Hadoop distributed processing technology, and further studied the implementation mechanism of the Metrics in Hadoop.Then,according to JMX and Metrics, a set of Hadoop monitoring system that can be applied to specific project is put forwared and perform well in practice.
\end{abstract}

\section{Keywords: JMX; Hadoop Monitor; J2EE}

\section{INTRODUCTION}

Hadoop is the most popular open source cloud computing based on distributed framework which can run on thousands of machines. In order to ensure reliability and robustness, we need to monitor hadoop's running status, health status, performance status. We can collect running information from cluster provided by Metrics system and JMX technology. Research on implementation mechanism of Metrics and JMX will help us to master the cluster health and reduce the failure rate in the project.

We can get monitor information through browser with default port provided by hadoop,but this kind of information can not applied to J2EE project. The default port provides a view of hadoop cluster for outside world, which is apparently not safe for the system. In the view of problem, the paper analysis the specific implementation of JMX in hadoop, and then design a monitor program written its monitoring and measurement functions in concrete project, at last realized Electronic document management system ( ERMS). The results showed the good effect of monitoring.

\section{THE TECHNICAL PRINCIPLE OF JMX}

Java Management Extensions(JMX)is java framework and specification for application management. It consists of a set of agents and services standards, which is committed to solvethe problems of distributed system management ${ }^{[1]}$.

The JMX technique is mainly divided into three layers: device layer, agent layer and distribution service layer. Device layer mainly defines the information model, The layer managed objects in the Mbean (Managed bean) exists in the form of management component. when need management it will be register for MBean server. The agent layer mainly defines the various services and communication model, the core component is MBean server, all the managed parts should register for it. Registered MBean does not directly communicate with remote program, they through the protocol adapter and connector for communication. Distributed service layer defines the main management interfaces and components,so that we can operate the managed components. the architecture are shown in figure 1:

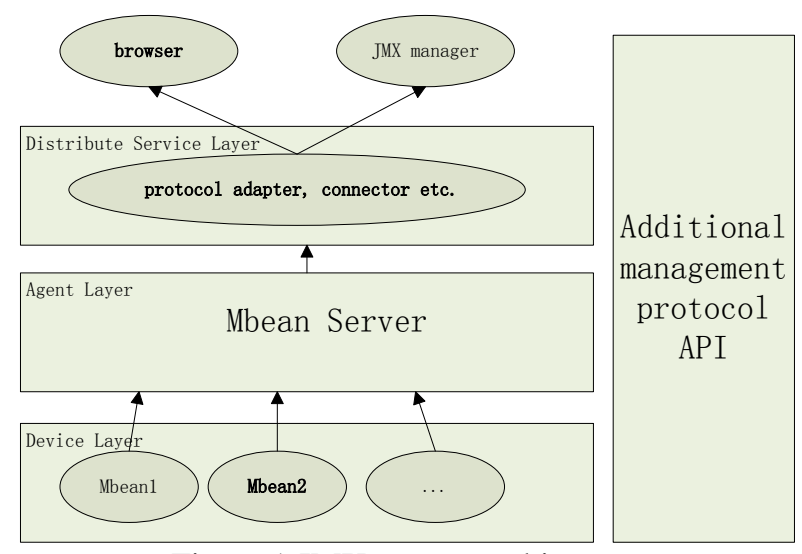

Fiugre 1 JMX system architecture

\section{A. Device Layer}

This layer implements the JMX manageable resources, including applications, network services, equipment etc.The core of layer is also MBean, which specifies the access attribute, the operate and how to send notification from MBean. There are four kinds of MBean in the system.

i) Standard MBeans: This type of MBean to use their own methods as management interface, Its design and realization is the most simple.

ii) Dynamic MBeans: It must achieve a specified Dynamic MBean interface, and the interface can be exposed and changed during runtime. and equipped with a data element such as Attribute, Opreator etc.

iii) Open MBeans: Make use of basic data types to achieve universal management, which belongs to dynamic Mbean.

iv) Model MBeans: It also belongs to dynamic Mbean, providing generic MBean class template for managed resources. which is fully configurable and could change itself during the operation. 
The communication among Managed resources MBean are requisite, JMX Notification plays a role of communication among MBean.

\section{B. Agent layer}

The layer manages Mbean resources provided by device layer and provides access for the remote user. Usually the agent layer compose of one MBean server and multiple system servers. Besides, communication protocol adapter and connector is also essential.

MBean server is the core component of the system, each MBean must be register for the MBean server and provide a unique object name format with "domain name: name=MBean",the name of domain and Mbean can be arbitrary.The agent layer could loading and unloading Mbean according to the need, so it has good scalability and flexibility.

\section{Distributed service layer}

The layer implements the interface of JMX application management platform, defines management interface and components that carry out operation for agent layer. The functions of components as follows: Provide an interface for managing application, so that it could interact with agent and JMX resource manager; By mapping the various agreements, provide a JMX agent and management component view; Construct a distributed system, release manage information to JMX agents;Collect manage information from JMX agents, filter the user interested information and form logic view according to the needs of end users. Besides, JMX also defines the Additional Management Protocol, Mainly used to support the current existing network management protocol, such as SNMP protocol, TMN protocol, CIM/WBEM protocol and so on.

\section{THE METRICS FRAMEWORK OF THE HADOOP}

\section{A. Hadoop introduction}

Hadoop is an open source distributed system architecture which belongs to Apache. It is mainly composed of HDFS (Hadoop Distributed File System), the composition of the MapReduce. Hadoop is a distributed computing platform for processing large-scale data parallel. The main advantages are: large-scale, low cost, efficient, safe, scalable etc.

HDFS is a distributed file system, which can be deployed in a cheap hardware, capable of high fault tolerance, mass data storage reliability. It not only provides shell command file for add, delete, copy, view ,upload and download based operation, but also provide the related file system access interface. In addition, also provide the function of disk load balancing, compression and decompression.

MapReduce is a programming model proposed by google,which can achieve large-scale parallel computing and processing massive amounts of data on a large scale distributed system server. This model is mainly composed of the core operation Map and Reduce. the flow shown in Figure 2.

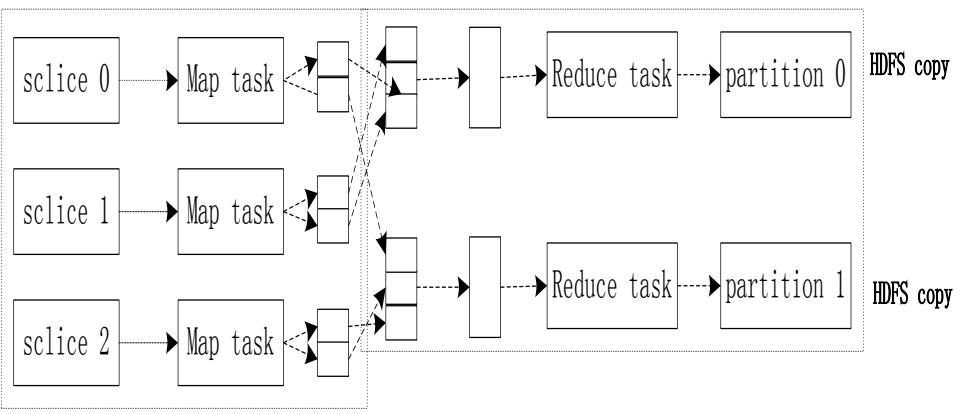

Figure2 Map/Reduce model implementation process

First the file data will be uploaded to the HDFS, then read the document file block (the default size of $64 \mathrm{MB}$ ) line by line and map the line number and content for the initial key value input, the Map function to separate the value part, to extract the keywords and the absolute file path , forming an intermediate key-value pair $<$ key, value $>$.

Reduce function is scheduled by the Master host, which as a input for the corresponding processing, generated by the Map function key-value pairs, the same key will be merge to the result of the output result $<$ key, value $>$. In the practical application, Map and Reduce can be free specified, that is brings great flexibility and the efficiency is very high. so it is very suitable for distributed search the large amount of data of simple data types.

\section{B. Hadoop Metrics}

Operation status, health status, performance status of Hadoop is mainly provided by its Metrics measurement system. In the diagram framework, Interface(MetricsSource) using getMetrics method to generate or update the metric. The method is driven by a timer thread. The MetricsSystem interface regularly pull measurement from the MetricsSource,MetricsSource allows the measurement tool to realize getMetrics method without thread lock. The putMetrics method provided by MetricsSinks interface is used to measure the immutable, so as not to cause the thread safety issues. MetricsFilter interface is used to measure the filtration generated by MetricsSource.

\section{APPLICATION OF JMX IN ERMS PROJECT BASED ON HADOOP}

\section{A. Introduction of ERMS project monitoring}

The template is designed so that author affiliations are not rep Eectronic document management system (ERMS), is mainly designed to manage the cycle of structured electronic documents, electronic documents, non structured entities. In system we store all files in hadoop HDFS, and process date by mapReduce. ERMS system administrator needs to know the basic status information of hadoop platform, for example, storage space, the file system state changes, whether in safe mode and other information, so the monitoring of Hadoop system is an important part of system management. Hadoop provides a simple webpage that get basic information of sytem,but this not good enough: 
(1)It is hard apply to specific J2EE project, and it is not easy for practical projects using Hadoop management;

(2) Security is low and default port number is known to the outside world。 Therefore expose the underlying file system of ERMS processing details;

(3) very simple, Only for HDFS and MapReduce, cannot monitor complex JVM measure. So we need a new solution to solve these problems.

Based on the research of JMX and Merics of the hadoop we can get to know, Through multiple metrics built by hadoop, data will be transformed to dynamic MBeans and register it to the JMX Mbean server. At last we could obtain and publish these data by using JMX framework.

\section{B. ERMS Monitoring design ideas}

\section{(1) Hadoop configuration file}

The premise of using the JMX monitoring Hadoop cluster is to enable remote access JMX function and set reasonable security level cluster, Including password authentication, SSL connection and a SSL client authentication etc.We can find master node, slave node, equalizer, jobtracker, tasktracker in conf/hadoop-env.sh.We can also set the value according to needs.

\section{(2) ERMS project configuration file}

JDK provides an easy to use tool Jconsole,it can get infromation of MBean. Through which we can of all kinds of information registry MBean, such as the ObjectName and its properties.Hadoop cluster using master-slave distributed structure. The NameNode and JobTracker node has only one, but DataNode and TaskTrakcker have multiple, we need to achieve the number of DataNode and TaskTrakcker in configuration file.

\section{(3) ERMS monitor interface design}

The ERMS project uses the MVC framework, monitoring information acquisition mainly work in the Model layer. The monitoring resources and interface framework shows in figure 3

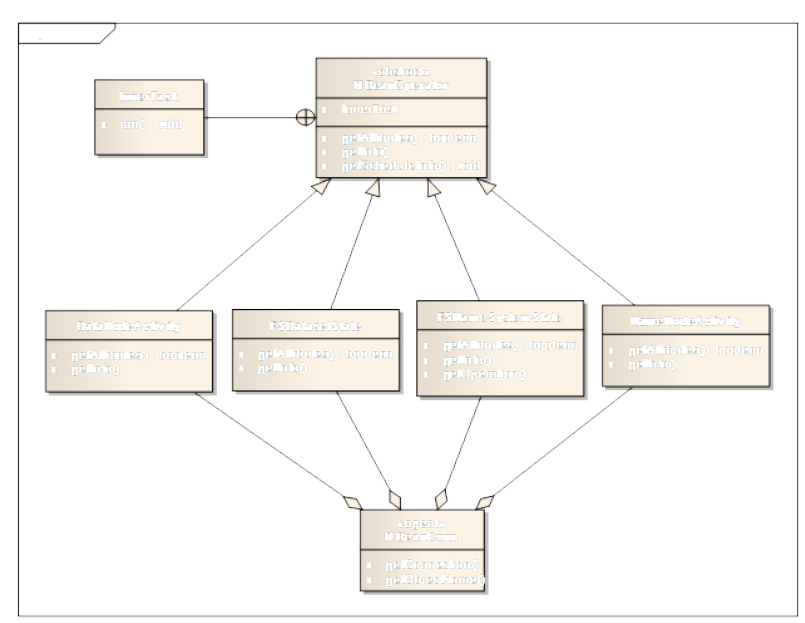

Figure3 hadoop framework in project
Access to information class and interface is mainly MBeanConn, MBeanOperator, InnerTask and MBean mapping of various classes of resources. MBeanConn class encapsulates the MBeanServer connection and get the ObjectName object operation, the connection address format using JMX specification format "/jndi/rmi://" + hostName + ":" + port + "/jmxrmi".MBeanOperator supply various abstract class which can be timed scheduling methods, so as to get the MBean information, the need of various MBean resource mapping class to inherit. MBean mapping of resource types, such as DataNodeActivity, the measurement of information does not meet the requirements of the project, the need for data processing, logic processing data is placed in the MBean resource mapping class. These MBean resources according to the project needs to expand.

\section{APPLICATION AND DISCUSSION}

The Hadoop cluster are made up of a management node and the two node data in the ERMS project, the metric structure from three nodes as shown below:

The information from background are need to be submitted to the web page, and then RestEasy module will handle the data, the technology can simplify web programming. The data through the Extjs to render the page, the metric information is displayed on the monitor interface.

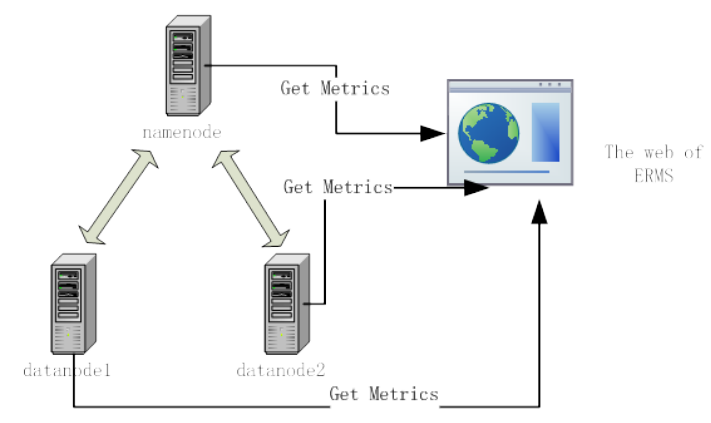

Figure 4 The actual control structure

The simply lists the JMX monitoring interface based on the HDFS distributed file system Hadoop, in fact, state information for MapReduce, JVM and RPC can also monitor

\section{CONCLUSION}

This paper studied the JMX specification and Metrics technical architecture of hadoop, put forward a kind of application of JMX technology to monitor the Hadoop cluster. This paper introduces the principle of design and specific for each step of the configuration operation, including files in the Hadoop cluster configuration, J2EE configuration, MBean project name to obtain, polling MBean in the project measurement code framework of information query. The plan proved feasibility through the application in specific projects. The work of the next step is to obtain more information of Hadoop running status, further improve the real-time monitoring. 


\section{REFERENCES}

[1] Bigtable: A Distributed Storage System for Structured Data. FAY CHANG 、 DEAN, JEFFREY , ACM Transactions on Computer Systems; Jun2008, Vol. 26 Issue 2

[2] Google MapReduce, Google, Inc., 2008

[3] The Google File System , Ghemawat,Sanjay;Leung, Shun-Tak , Operating Systems Review, 2003

[4] Google's MapReduce programming model — Revisited , Ralf Lämmel, Science of Computer Programming, Volume 70, 2008

[5] Y. Yorozu, M. Hirano, K. Oka, and Y. Tagawa, "Electron spectroscopy studies on magneto-optical media and plastic substrate interface,” IEEE
Transl. J. Magn. Japan, vol. 2, pp. 740-741, August 1987 [Digests 9th Annual Conf. Magnetics Japan, p. 301, 1982].

[6] M. Young, The Technical Writer's Handbook. Mill Valley, CA: University Science, 1989.

[7] IBM, "Big Data at the speed of business," http://www-01.ibm.com/ software/data/bigdata/what-is-big-data.html,

[8] J. Dean and S. Ghemawat, "MapReduce: simplified Data processing on large clusters," Communications of the ACM, Vol. 51, no. 1, pp. 107113, 2008. 